\title{
3 Research Square

\section{Modified Posterior Short-Segment Pedicle Screw Fixation at The Fracture Level for Treatment of Upper Lumbar Burst Fracture}

\section{Xubiao Chen}

Shantou Central Hospital

Shixin Lu

Sun Yat-Sen Memorial Hospital

Songjie Yang

Shantou Central Hospital

Junshen Huang

Sun Yat-Sen Memorial Hospital

Jiajun Huang

Sun Yat-Sen Memorial Hospital

Yuwei Liang

Sun Yat-Sen Memorial Hospital

Xiangge Liu

Sun Yat-Sen Memorial Hospital

Ziying Cheng

Sun Yat-Sen Memorial Hospital

Yuxi Li

Sun Yat-Sen Memorial Hospital

Lin Huang ( $\square$ huangl5@mail.sysu.edu.cn)

Sun Yat-Sen Memorial Hospital https://orcid.org/0000-0002-8285-2658

Research article

Keywords: Burst fracture, Short-segment fixation, Lumbar, Modified technique, Posterior

Posted Date: December 18th, 2020

DOI: https://doi.org/10.21203/rs.3.rs-129857/v1

License: (1) (1) This work is licensed under a Creative Commons Attribution 4.0 International License.

Read Full License 


\section{Abstract}

\section{Introduction}

Upper lumbar burst fractures (L1or L2) are often followed by bilateral pedicle screw fixation at the level of fracture using posterior short-segment pedicle instrumentation. However, it can aggravate the trauma to the fractured vertebra. We have introduced a modified technique of posterior short-segment instrumentation for the treatment of upper lumbar burst fracture. The aim of this study was to compare the clinical and radiologic results of modified technique versus conventional technique using posterior short-segment pedicle instrumentation in the treatment of upper lumbar burst fractures.

\section{Methods}

The data from 64 patients with upper lumbar burst fracture who had undergone posterior short-segment instrumentation from April 2014 to November 2018 in our clinic were evaluated in the present retrospective study. All the patients were divided into 2 groups according to the surgical technique, including 27 patients (modified order of intraoperative pedicle screw placement) in modified group and 37 patients (conventional order of intraoperative pedicle screw placement) in conventional group. The clinical outcomes and radiological parameters were evaluated preoperatively, postoperatively, at 3-month follow-up and final follow-up.

\section{Results}

Technical success was achieved in all 64 patients. The operation time of modified group $(130.4 \pm 32.4 \mathrm{~min})$ is significantly longer than conventional group $(115.3 \pm 26.8 \mathrm{~min}, p<0.05)$. Significant improvement in the anterior vertebral height $(\mathrm{AVH})$ ratio $(97.9 \% \pm 6.7 \%$ in modified group, $94.1 \% \pm 7.8 \%$ in conventional group, $p<0.05)$ was found postoperatively, $(100.1 \% \pm 9.7 \%$ in modified group, $89.6 \% \pm 6.7 \%$ in conventional group, $p<0.01)$ at the 3 -month follow-up and $(98.8 \% \pm 7.7 \%$ in modified group, $90.9 \% \pm 7.6 \%$ in conventional group, $p<0.01)$ at the final follow-up. And post-operative correction of $\mathrm{AVH}$ ratio was significantly better in modified group (45.0\%) than in conventional group $(38.8 \%, p<0.01)$. There was 1 case of wound infection in both groups respectively. No instrument loosening or failure, or breakage was observed during followup.

\section{Conclusions}

Modified technique and conventional technique of posterior short-segment pedicle screw fixation for upper lumbar burst fractures both provided immediate stability and reduction of post-traumatic segmental kyphosis. In addition, modified technique of posterior short-segment pedicle screw fixation seems to be a promising method for upper lumbar burst fractures because it led to better reduction of fractured vertebra than in patients who received conventional technique.

\section{Introduction}


Most traumatic thoracolumbar fractures occur at the thoracolumbar junction (T11-L2) ${ }^{[1]}$. Operative treatment should be considered when anterior vertebral body height loss exceeds $50 \%$, when the spinal canal is compromised by more than $50 \%$, or when angulation is greater than $20^{\circ}[2]$. Recently, Thoracolumbar Injury Classification and Severity(TLICS) score which took fracture morphology, neurological injury and the integrity status of posterior ligamentous into account has been applied to assess spinal stability and determine operative or nonoperative treatment ${ }^{[3]}$. According to this classification, surgical intervention is suggested for a score $\geq 5$ points, and conservative nonoperative treatment for a score $\leq 3$ points ${ }^{[3 ; 4]}$. Currently the right approach and instrumentation techniques continue to be controversial ${ }^{[5]}$. Nowadays, several surgical techniques, including posterior, anterior, open, minimally invasive, and combined posterior-anterior ones, are available for the treatment of thoracolumbar burst fractures.

In recent years, short-segment pedicle screws fixation (inclusion of one level above and one level below the fractured vertebra) offers the advantage of preserving motion of the spinal segments, a shorter operative time, less blood loss, and less invasiveness so that it has become the most commonly surgical technique ${ }^{[6 ; 7]}$. However, disadvantages of this method, such as high incidence of early instrumentation failure, and increases in kyphosis, have been reported ${ }^{[8 ; 9]}$. To prevent these failures, some surgeons suggested adding pedicle screws at the level of the fractured vertebra ${ }^{[5 ; 10]}$. Gradually, it is confirmed that this technique has better outcomes than patients with short-segment pedicle instrumentation. Its advantages of increasing the stiffness of the construct and protecting the anterior column also have been demonstrated by biomechanical studies ${ }^{[10 ; 11]}$. However, some groups have suggested that the pedicle screw placement at the fracture level aggravate the trauma to the fractured vertebra. Therefore, how to improve the curative effect of this technique is worthy of further study. Upper lumbar(L1-L2) burst fractures are the most common injuries to the lumbar spine after compression fractures. In this study, we aimed to compare the clinical and radiologic results of two techniques of posterior short-segment pedicle screw fixation for treatment of upper lumbar burst fracture.

\section{Materials And Methods}

\section{Subjects}

From April 2014 to November 2018, 64 patients with acute, traumatic fractures of upper lumbar junction (L1 or L2) have been treated with posterior pedicle screw fixation and fusion in our department. The inclusion criteria for subjects were as follows: age between 18 and 60 years; single-level upper lumbar(L1or L2) fracture; Thoracolumbar Injury Classification and Severity score $\geq 4$; fracture duration $<1$ weeks囚application of posterior short-segment pedicle screw instrumentation at the fracture level. Our exclusion criteria were as follows: multisegmental lumbar fracture; Thoracolumbar Injury Classification and Severity score $\mathbb{4}$ \long segment instrumentation; and pathological fractures. A written informed consent was obtained from each patient before the surgical procedures for the use of their personal 
information for research purposes. The patients were divided into two groups according to the surgical technique of posterior short-segment pedicle screw fixation.

\section{Clinical and radiographic parameters}

We had evaluated the age, gender, trauma etiology, fracture type, fracture level, and clinical and radiologic outcomes of patients in two groups. The anteroposterior and lateral radiographs, computed tomography (CT) and magnetic resonance imaging (MRI) scans of lumbar spine were taken for all the patients. We classify the fracture type and to estimate the severity of the fracture by CT. And we verified whether the posterior ligamentous complex and spinal cord were damaged according to MRI. We use the thoracolumbar injury classification system ${ }^{[12]}$ to evaluate the operative indication. The results were compared in a retrospective, nonrandomized cohort study.

Radiologic parameters were assessed from the plain radiographs including local kyphosis angle (LKA), and anterior vertebral height $(\mathrm{AVH})$ and their mean value was quantified. The ratio of the AVH was determined using the following formula: $\mathrm{AVH}$ ratio $=\mathrm{AVH} /[$ (superior vertebra $\mathrm{AVH}+$ inferior vertebra AVH)/2] $\times 100$ (Figure 1A). The local kyphosis angle (LKA) was measured between the superior and inferior endplates of the injured vertebra using the Cobb method (Figure 1B) on the lateral radiograph. Data for preoperative, immediate post-operative and follow-up radiological measures were collected. We use Visual Analog Scale (VAS) scores to assess the clinical outcomes. We evaluated the presence of implant failure including implant loosening, screw pullout, screw breakage, rod breakage, and an increase in the LKA of more than $10^{\circ}$ according to the follow-up radiographs ${ }^{[13]}$.

\section{Statistical analysis}

The software Statistical Package for the Social Sciences (SPSS, version 25.0) was used for the statistical analysis of the data. Continuous variables are expressed as the mean \pm standard deviation. Categorical variables are presented as numbers and/or percentages. Independent $t$ tests were employed for the comparison of continuous variables between groups and Pearson $\chi 2$ or the Fisher exact test were employed in the comparison of categorical variables between groups. Any value of $p$-value $<0.05$ were considered significant.

\section{Surgical technique}

All the patients were in the prone position. Under general anesthesia, posterior midline incision was performed. The fracture level was generally identified by C-arm. Modified technique of posterior shortsegment instrumentation was performed as follows: four pedicle screws are first inserted into the vertebrae one level above and below the fractured vertebra. Distraction is performed longitudinally on one side using spreader forceps to restore lordosis and body height after connecting the rods and screws. Then we temporarily fix the titanium rod of the distracted side, remove the titanium rod and insert one 
pedicle screw into the fractured vertebra on the contralateral side. Next, we replace the rod, distract lengthwise and fix it. After that we remove the titanium rod on the previously distracted side, insert one pedicle screw into the fractured vertebra and replace the rod. Finally we distract again and tighten adjacent fractured vertebra screws nuts before we tighten the fractured vertebra screws nuts (Figure 2) and install the cross link. Conventional technique of posterior short-segment instrumentation was performed as follows: a total of six pedicle screws are inserted into the fractured vertebra and one level above and below the fractured vertebra. After connecting the rods and screws, distraction is performed to correct the deformity and achieve indirect decompression. The instrumentation is tightened by screw nuts (Figure 3 ) and cross link finally. After irrigation, the incision is closed in layers over the drain.

Decompressive laminectomy was only performed in one patient of modified group with severe spinal canal compression in pre-operative CT and MRI. Bone grafts of the injured vertebrae and vertebroplasty were not conducted in all patients. The degree of kyphosis correction and signs of implant failure (such as screw breakage, rod displacement/breakage, screw head dislodgement) were assessed by the postoperative radiographs. More evaluated parameters were operative time and surgical complications.

\section{Results}

\section{Patient demographics}

This study contained a total of 64 patients, including 37 males and 27 females. Modified group included 27 patients with the average age of 43.0 years (range: 26-60 years). This group contained 18 males and 9 females. Conventional group included 37 patients with the average age of 45.7 years (range: 29-59 years), which is consisted of 19 males and 18 females. No statistically difference was discovered between the two groups in age and gender $(p>0.05)$. All the patients in this study got the TLICS scores of equal to or more than 4 points, which suggests operative treatment according to the Thoracolumbar Injury Classification and Severity score (TLICS). As for lumbar fracture level in modified group, 20 patients were in L1 and 7 in L2. And in the conventional group, 21 in L1 and 16 in L2. The high falling was the most common injury mechanism in both groups, followed by the road traffic accident. There were no significant differences for TLICS scores, fracture level and mechanism of injury $(p>0.05)$. There was one wound infection in each group respectively. All these complications were treated conservatively with complete resolution. No other serious complications such as blood vessel injury, spinal cord or nerve root injury related to surgery were observed in both groups. In all cases, fusions healed well and no revision surgery was performed for loss of correction or failure of instrumentation during follow-up.

Table 1 Patient demographic and injury details 


\begin{tabular}{|c|c|c|c|}
\hline Index & Modified group & Conventional group & $p$ value \\
\hline Number of patients & 27 & 37 & \\
\hline Mean age(year) & 43.0 & 45.7 & 0.223 \\
\hline \multicolumn{4}{|l|}{ Gender } \\
\hline Male & 18 & 19 & \multirow[t]{2}{*}{0.220} \\
\hline Female & 9 & 18 & \\
\hline \multicolumn{4}{|l|}{ Mechanism of injury } \\
\hline Vehicle accident & 1 & 2 & \multirow[t]{2}{*}{1.000} \\
\hline Falling & 26 & 35 & \\
\hline \multicolumn{4}{|l|}{ Level of fracture } \\
\hline L1 & 20 & 21 & \multirow[t]{2}{*}{0.154} \\
\hline L2 & 7 & 16 & \\
\hline \multicolumn{4}{|l|}{ TLICS score } \\
\hline 4 & 8 & 19 & \multirow[t]{5}{*}{0.280} \\
\hline 5 & 5 & 6 & \\
\hline 6 & 2 & 0 & \\
\hline 7 & 5 & 6 & \\
\hline 8 & 7 & 6 & \\
\hline \multicolumn{4}{|c|}{ Surgical complications } \\
\hline Wound infection & 1 & 1 & 1.000 \\
\hline
\end{tabular}

\section{Comparison of clinical data}

As shown in Table 2, the mean operative time in modified group is significantly longer than conventional group (130.4 $\pm 32.4 \mathrm{~min}$ in modified group, $115.3 \pm 26.8 \mathrm{~min}$ in conventional group, $p=0.046$ ). The total volume of blood loss was $290.7 \pm 186.1 \mathrm{ml}$ in the modified group and $255.4 \pm 209.1 \mathrm{ml}$ in the conventional group, which was not statistically significant $(p=0.487)$. The postoperative drainage was $261.9 \pm 153.4 \mathrm{ml}$ in the modified group and $292.9 \pm 147.5 \mathrm{ml}$ in the conventional group, which was also not statistically significant $(p=0.417)$. Also, the VAS scores between two groups were similar for both groups $(p>0.05)$ (Table 2).

Table 2 Operation values and VAS score 


\begin{tabular}{|llll|}
\hline Operative Characteristic & Modified group & Conventional group & $\boldsymbol{p}$ value \\
\hline Operating time $(\mathrm{min})$ & $130.4 \pm 32.4$ & $115.3 \pm 26.8$ & 0.046 \\
\hline Blood loss $(\mathrm{ml})$ & $290.7 \pm 186.1$ & $255.4 \pm 209.1$ & 0.487 \\
\hline Postoperative drainage $(\mathrm{ml})$ & $261.9 \pm 153.4$ & $292.9 \pm 147.5$ & 0.417 \\
\hline VAS score & & & \\
\hline Pre-operative & $7.4 \pm 1.0$ & $7.2 \pm 1.2$ & 0.581 \\
\hline Post-operative & $4.2 \pm 1.3$ & $4.1 \pm 1.1$ & 0.966 \\
\hline Last follow-up & $2.0 \pm 1.3$ & $2.0 \pm 1.0$ & 0.972 \\
\hline
\end{tabular}

\section{Comparison of radiological data}

\section{Local kyphosis angle (LKA)}

The average preoperative LKA was $13.8^{\circ} \pm 7.2^{\circ}$ in modified group and $10.7^{\circ} \pm 5.5^{\circ}$ in conventional group, which was significantly corrected to $4.7^{\circ} \pm 4.6^{\circ}$ and $4.1^{\circ} \pm 3.8^{\circ}$ (all $p<0.05$ ) after surgery, respectively. Postoperative correction was slightly better in modified group (66.9\%) than in conventional group (64.5\%) without no statistical relevance $(p=0.725)$. There was a loss (mean $2.5^{\circ}$ in modified group and $4.5^{\circ}$ in conventional group) in the final follow-up, resulting in a mean LKA of $6.1^{\circ} \pm 6.2^{\circ}$ and $8.8^{\circ} \pm 5.1^{\circ}$, respectively. Correction loss in modified group was slightly less than in conventional group. However, this difference was also not statistically significant $(p=0.186)$. These results are summarized in Table 3 . To conclude, the two groups were similar both in terms of post-operative sagittal alignment restoration and correction loss at last follow-up.

\section{Anterior vertebral height (AVH)ratio of the fractured vertebra}

Significant improvement in the $\mathrm{AVH}$ ratio $(97.9 \% \pm 6.7 \%$ in modified group, $94.1 \pm 7.8$ in conventional group, $p=0.043)$ was found postoperatively, $(100.1 \% \pm 9.7 \%$ in modified group, $89.6 \% \pm 6.7 \%$ in conventional group, $p=0.001)$ at the 3 -month follow-up and $(98.8 \% \pm 7.7 \%$ in modified group, $90.9 \% \pm 7.6 \%$ in conventional group, $p=0.002$ ) at the final follow-up. Post-operative correction was significantly better in modified group $(45.0 \%)$ than in conventional group $(38.8 \%, p=0.007)$. There was a loss (mean $0.6 \%$ in modified group and $2.6 \%$ in conventional group) in the final follow-up. Correction loss in modified group was slightly less than in conventional group without no statistical relevance $(p=0.227)$. Changes of the AVH ratio, which reflect the fracture-induced wedge shape of the vertebral body, are presented in Table 3 . To conclude, modified surgical method resulted in a better reconstruction of the original shape of the vertebral body than conventional surgical method postoperatively. However, they shown no significant difference in correction loss at last follow-up. Figure 4 shows a representative case in the modified group.

Table 3 Comparison of radiological data between groups 


\begin{tabular}{|llll|}
\hline & Modified group & Conventional group & $p$ value \\
\hline Local kyphosis angle (LKA) & & & \\
\hline Pre-operative & $13.8 \pm 7.2$ & $10.7 \pm 5.5$ & 0.060 \\
\hline Post-operative & $4.7 \pm 4.6$ & $4.1 \pm 3.8$ & 0.574 \\
\hline Follow-up at 3 months & $5.7 \pm 4.4$ & $8.0 \pm 4.7$ & 0.147 \\
\hline Last follow-up & $6.1 \pm 6.2$ & $8.8 \pm 5.1$ & 0.116 \\
\hline Correction loss & $2.5 \pm 5.4$ & $4.5 \pm 4.5$ & 0.186 \\
\hline Correction (\%) & $66.9 \pm 27.1$ & $64.5 \pm 26.2$ & 0.725 \\
\hline Anterior vertebral & & & \\
\hline height ratio (\%) & & & 0.263 \\
\hline Pre-operative & $52.9 \pm 10.0$ & $55.2 \pm 6.7$ & 0.043 \\
\hline Post-operative & $97.9 \pm 6.7$ & $94.1 \pm 7.8$ & 0.001 \\
\hline Follow-up at 3 months & $100.1 \pm 9.7$ & $89.6 \pm 6.7$ & 0.002 \\
\hline Last follow-up & $98.8 \pm 7.7$ & $90.9 \pm 7.6$ & 0.227 \\
\hline Correction loss & $0.6 \pm 4.5$ & $2.6 \pm 6.5$ & 0.007 \\
\hline Correction & $45.0 \pm 9.6$ & $38.8 \pm 8.2$ & \\
\hline
\end{tabular}

\section{Neurological outcome and complications}

Based on muscle strength classification, at admission 13 patients (48.15\%) had Grade V muscle strength; five patients (18.52\%) had Grade IV muscle strength, and nine patients (33.33\%) had Grade I to III muscle strength in modified group. Follow-up muscle strength was evaluated as following: Grade $V$ muscle strength (21 patients: $77.78 \%$ ), Grade IV muscle strength (three patients: $11.11 \%$ ), and Grade I to III muscle strength (three patients: $11.11 \%)$. In a word, 14 patients out of $27(51.9 \%)$ showed muscle strength improvement (Table 4).

As for conventional group, 28 patients (75.68\%) had Grade $V$ muscle strength; seven patients $(18.92 \%)$ had Grade IV muscle strength, and two patients $(5.41 \%)$ had Grade I to III muscle strength at admission. At follow-up, 34 patients (91.89) had Grade V muscle strength and 3 patients (8.11\%) Grade IV muscle strength. In conventional group, seven patients out of 37 (18.92\%) showed muscle strength improvement (Table 4). Patients with incomplete paralysis in the modified group were more than those in conventional group before operation, while statistical analysis showed no difference in postoperative muscle strength between the two groups $(p>0.05)$. 
In modified group, at admission eight patients (29.63\%) had dysuria and four patients $(14.81 \%)$ still had dysuria after operation, which means four patients out of $27(14.81 \%)$ showed neurological improvement (Table 4). In conventional group, at admission eight patients (21.62\%) had dysuria and one patient (2.70\%) still had dysuria postoperatively, which means seven patients out of 37(18.92\%) showed neurological improvement (Table 4). Statistical analysis showed no difference between the two groups ( $p$ $>0.05$ ).

Complications included one urinary infection and two deep wound infection, one in each group. All these complications were treated conservatively with complete resolution. What's more, instrumentation failure such as bending, loosening, or breakage of the pedicle screws was not observed in any patient during the follow-up period.

Table 4 Comparison of Neurological outcome and complications between groups

\begin{tabular}{|c|c|c|c|}
\hline & Modified group & Conventional group & $p$ value \\
\hline \multicolumn{4}{|c|}{ Preoperative muscle strength } \\
\hline I-III & 9 & 2 & \multirow[t]{3}{*}{0.011} \\
\hline IV & 5 & 7 & \\
\hline V & 13 & 28 & \\
\hline \multicolumn{4}{|c|}{ Postoperative muscle strength } \\
\hline $\mid-I I I$ & 3 & 0 & \multirow[t]{3}{*}{0.117} \\
\hline IV & 3 & 3 & \\
\hline V & 21 & 34 & \\
\hline Preoperative dysuria & 8 & 8 & 0.563 \\
\hline Postoperative dysuria & 4 & 1 & 0.153 \\
\hline \multicolumn{4}{|l|}{ Complications } \\
\hline Deep wound infection & 1 & 1 & 1.000 \\
\hline Urinary infection & 0 & 1 & 1.000 \\
\hline
\end{tabular}

\section{Discussion}

The optimal treatment for thoracolumbar burst fractures is controversial ${ }^{[14]}$. The purpose of operation is to relieve the compression caused by fracture block and provide reliable spine stability. The operation approach includes anterior approach, posterior approach and combined anterior and posterior approach. Each of them has its own advantages and disadvantages, and there is still controversy over the approach selection. Posterior operation utilizing the tension of the posterior longitudinal ligament to restore the 
fracture block protruding in spinal canal has the advantages of easier exposure and operation, less trauma, shorter operation time, less bleeding, and better three-dimensional fixation so on ${ }^{[15]}$. Its superiority is also confirmed by the study of Stancić et al[ ${ }^{[16]}$. In recent years, it is the most mainstream operation for thoracolumbar burst fractures in many hospitals. Waqar et al ${ }^{[17]}$ also considered that posterior surgery is one of the most effective methods for the treatment of thoracolumbar burst fractures. Although posterior long-segment pedicle screw fixation enhanced spinal stability, it sacrificed the range of activity of the spine, which may lead to low back stiffness, chronic pain and so on. However, posterior short-segment pedicle screw fixation can not only maintain spinal stability and restore the height of fractured vertebra but also can retain more spinal motion segments ${ }^{[18]}$. So the posterior short -segment pedicle screw fixation is the mainstream technique for single segment vertebra burst fracture. body. In this study, all patients were single upper lumbar burst fracture treated with posterior short-segment pedicle screw fixation.

After internal fixation, when the spine is moving, it can produce a certain force of torque to the screw-rod system, and the placement of the screw through the fractured vertebra can make the screws of the fractured vertebra bear a certain amount of stress, which reduces the stress of the screws of the adjacent superior and inferior vertebra ${ }^{[19]}$. The fractured vertebral screws divide the screw-rod fixation into two parts, which shortens the torque, reduces the load of screw-rod stress to a certain extent and reduces the failure rate of internal fixations. What's more, the placement of screws through fractured vertebra can improve the stress distribution of adjacent screws and reduce the stress of screws, thus it can reduce the rate of screws breakage ${ }^{[20]}$. Meta-analysis showed that although trans fractured vertebral screws placement may lead to more blood loss and more operation time, the technique of screws placement through fractured vertebra had more advantages in maintaining spinal height and flexion than the technique without fractured vertebra screws ${ }^{[21]}$. It has a better effect on the reconstruction of spinal physiological sequence and the restoration of spinal stability.

Long-term follow-up suggested that the main complications of short segment pedicle screw fixation system in the treatment of thoracolumbar burst fracture was the loss of correction and internal fixation failure caused by the decrease of vertebral body height and intervertebral space stenosis ${ }^{[22]}$. When severe burst fracture occurs to the lumbar vertebra, it can produce many fracture blocks causing the overlapping and compression of fracture blocks. In conventional technique of posterior short-segment pedicle instrumentation, single distraction and reduction during the operation may be difficult to complete because of the mutual compression of the fracture blocks, especially when the pedicle screws of fractured vertebra have been inserted firstly. The obstruction of the screws results in poor reduction of the fracture block during distraction. At the same time, the swing and cutting effect of the screws of fractured vertebra in the distraction of the adjacent vertebra (Figure. 5) results in further compression of part of the bone. It may lead to larger bone defects. In modified technique of posterior short-segment pedicle instrumentation, repeated distraction and reduction and continuous traction make the soft tissue around the fracture relax, which is beneficial for the reduction of fracture blocks. And the compression of anterior 
and posterior longitudinal ligament caused indirect reduction of fracture blocks. What's more, centripetal traction of annulus fibrousus can also promote the reduction of fracture blocks.

When the pedicle screw-rod system is used for distraction, the cortical bone and endplate on the surface of the vertebral body can be reduced under the traction of the anterior and posterior longitudinal ligament and the fibrous annulus of intervertebral disc, while the compressed cancellous bone in the vertebral body cannot be re-expanded, resulting in bone defect ${ }^{[23]}$. Insufficient strength of vertebral body makes itself easy to be compressed again, resulting in the loss of correction. Some scholars have put forward the theory of injured vertebra reinforcement, that is, bone grafting into the vertebral body through pedicle can strengthen the stability of the anterior and middle column of spine and reduce the long-term complications such as fracture and loosening of internal fixation and the loss of fractured vertebral height ${ }^{[24]}$. However, Alanay et al ${ }^{[13]}$ and Knop et al ${ }^{[25]}$ believed that transpedicular bone grafting could not prevent the collapse of vertebral body and the loss of correction. Some studies even showed that the reduction height of fractured vertebra in bone grafting group was higher than that in non-bone grafting group. In this study, the patients in modified group can avoid the further bone compression caused by the swing of the injured vertebral screw by the distraction before fractured vertebra screws placement. At the same time, it can drive the posterior fracture block to move forward and fill the defect in the vertebral body, which can not only achieve a better reduction effect, but also reduce the long-term bone defect, so as to reduce the loss of correction.

Both open reduction and manual reduction of limb fractures require continuous longitudinal traction to relax the muscles around the fracture area and then push the end of the long bone to promote better reduction of the fracture (Fig. 5). The modified technique uses longitudinal traction firstly to make use of posterior longitudinal ligament to restore, which is equivalent to longitudinal force. The placement of fractured pedicle screws is equivalent to the transverse force, which pushes the fracture block forward (Figure. 6). We skillfully apply the longitudinal and transverse force to achieve the effect of threedimensional reduction, which can get a better reduction. At the same time, the fracture blocks were shifted forward to fill the anterior bone defect, which is better to prevent the loss of reduction. In the practice of modified pedicle screws placement, we have accumulated a lot of experience. According to the situation of nerve injury and the results of $\mathrm{CT}$ before operation, we distract the adjacent vertebra firstly on the seriously fractured side (such as the left side), then place the fractured pedicle screw on the mildly fractured side (right side). After multiple distraction, we insert the pedicle screw on the severe side (left side). Combined with the reduction effect shown by the $\mathrm{X}$-ray fluoroscopy during the operation, we can learn the reduction of the posterior edge of the vertebral body. If the reduction is not good, we distract repeatedly during operation to improve reduction effect, and even employ over-distraction and temporary fixation to promote the reduction of the fracture blocks. If the reduction is still not effective, laminectomy and spinal canal decompression should be considered.

In the patients with vertebra burst fracture complicated with intervertebral disc injury, the nucleus of the intervertebral disc was pressed by the endplate embedded in the vertebral body. The pedicle screw-rod system can restore the anterior and posterior wall and the height of the vertebral body through the 
distraction of the ligament, but it cannot restore the central region of the collapsed endplate, so the intervertebral disc can trap into this area. The loss of water in the nucleus of the fractured intervertebral disc is accelerating. And the injury to the endplate can directly affect the nutrition supply of the intervertebral disc, which will accelerate the intervertebral disc degeneration and leads to the stenosis of the intervertebral space and the loss of correction ${ }^{[26]}$. In this study, the anterior vertebral height (AVH) ratio of modified group on postoperation, follow-up at 3 months and last follow-up were respectively $0.98 \pm 0.07,0.99 \pm 0.07,0.96 \pm 0.11$, which is significantly higher than those of conventional $\operatorname{group}(p<0.05)$. We believe repeated distraction can better promote the rearrangement of fracture blocks and continuous traction before the placement of fractured vertebra screws can better promote the reduction of incarcerated intervertebral disc in modified group so as to improve the intervertebral space and reduce the long-term loss of correction. It's also the reason why the operation time of modified group is longer than that of conventional group, with no significant difference in the results of blood loss and drainage volume between two groups.

This retrospective study had some limitations because it was a small, single-center, nonrandomized study. Keeping these limitations in mind and based on clinical outcomes, the use of modified technique of posterior short-segment pedicle instrumentation is a valuable treatment option for upper lumbar burst fractures. However, prospective and randomized studies with more patients and longer follow-up are needed to draw definitive and accurate conclusions.

\section{Conclusions}

Modified technique and conventional technique of posterior short-segment pedicle instrumentation for the treatment of upper lumbar burst fractures both provided immediate stability and reduction of posttraumatic segmental kyphosis. In addition, modified technique of posterior short-segment pedicle instrumentation seems to be a promising method for upper lumbar burst fractures because it led to better reduction of fractured vertebra than in patients who received conventional technique.

\section{Abbreviations}

Thoracolumbar Injury Classification and Severity (TLICS)

local kyphosis angle (LKA)

anterior vertebral height $(\mathrm{AVH})$

\section{Declarations}

Ethics approval and consent to participate: A written informed consent was obtained from each patient before the surgical procedures for the use of their personal information for research purposes.

Consent for publication: All authors have approved the version submitted for publication. 
Availability of data and materials: All the data and materials of this article are valid and authentic.

Competing interests: All the authors of this article confirm that no author has any conflict of interest to disclose.

Funding: This study was supported by the National Natural Science Foundation of Guangdong Province (grant no. 2017A030310554, 2020A1515010371 and 2017A030313652).

Authors' contributions: Xubiao Chen and Shixin Lu: study concept and design, interpretation of data, and composition of the manuscript. Songjie Yang: analysis and interpretation of data. Junshen Huang and Jiajun Huang: acquisition of data and composition of the manuscript. Yuwei Liang: acquisition of data and supervision of the manuscript. Xiangge Liu and Ziying Cheng: analysis and interpretation of data and composition of the manuscript. Lin Huang and Yuxi Li: study concept and design, analysis and interpretation of data, and composition of the manuscript..

Acknowledgements: This study was supported by the National Natural Science Foundation of Guangdong Province (grant no. 2017A030310554, 2020A1515010371 and 2017A030313652).

\section{References}

1. Ruiz Santiago, F., Tomas Munoz, P., Moya Sanchez, E., Revelles Paniza, M., Martinez Martinez, A., \& Perez Abela, A. L. (2016). Classifying thoracolumbar fractures: role of quantitative imaging. Quantitative imaging in medicine and surgery, 6(6), 772-84. doi:10.21037/qims.2016.12.04

2. Benson, D. R., Burkus, J. K., Montesano, P. X., Sutherland, T. B., \& McLain, R. F. (1992). Unstable thoracolumbar and lumbar burst fractures treated with the AO fixateur interne. Journal of spinal disorders, 5(3), 335-43.

3. Patel, A. A., Dailey, A., Brodke, D. S., Daubs, M., Harrop, J., Whang, P. G., \& Vaccaro, A. R. (2009). Thoracolumbar spine trauma classification: the Thoracolumbar Injury Classification and Severity Score system and case examples. J Neurosurg Spine, 10(3), 201-6. doi:10.3171/2008.12.SPINE08388

4. Hitchon, P. W., Abode-lyamah, K., Dahdaleh, N. S., Shaffrey, C., Noeller, J., He, W., \& Moritani, T. (2016). Nonoperative Management in Neurologically Intact Thoracolumbar Burst Fractures: Clinical and Radiographic Outcomes. Spine, 41(6), 483-9. doi:10.1097/BRS.0000000000001253

5. Kose, K. C., Inanmaz, M. E., Isik, C., Basar, H., Caliskan, I., \& Bal, E. (2014). Short segment pedicle screw instrumentation with an index level screw and cantilevered hyperlordotic reduction in the treatment of type-A fractures of the thoracolumbar spine. The bone \& joint journal, 96-B(4), 541-7. doi:10.1302/0301-620X.96B4.33249

6. Pellisé, F., Barastegui, D., Hernandez-Fernandez, A., Barrera-Ochoa, S., Bagó, J., Issa-Benítez, D., . . . Villanueva, C. (2015). Viability and long-term survival of short-segment posterior fixation in thoracolumbar burst fractures. The spine journal : official journal of the North American Spine Society, 15(8), 1796-803. doi:10.1016/j.spinee.2014.03.012 
7. Sun, C., Guan, G., Liu, X., Zhang, H., \& Wang, B. (2016). Comparison of short-segment pedicle fixation with versus without inclusion of the fracture level in the treatment of mild thoracolumbar burst fractures. International journal of surgery (London, England), 36(Pt A), 352-7.

doi:10.1016/j.ijsu.2016.11.086

8. Korovessis, P. G. (2007). Re: Mahar A, Kim C, Wedemeyer M, et al. Short-segment fixation of lumbar burst fractures using pedicle fixation at the level of the fracture. Spine 2007;32:1503-7. Spine, 32(23).

9. Xu, B.-s., Tang, T.-S., \& Yang, H.-I. (2009). Long-term results of thoracolumbar and lumbar burst fractures after short-segment pedicle instrumentation, with special reference to implant failure and correction loss. Orthop Surg, 1(2), 85-93. doi:10.1111/j.1757-7861.2009.00022.x

10. Guven, O., Kocaoglu, B., Bezer, M., Aydin, N., \& Nalbantoglu, U. (2009). The use of screw at the fracture level in the treatment of thoracolumbar burst fractures. Journal of spinal disorders \& techniques, 22(6), 417-21. doi:10.1097/BSD.0b013e3181870385

11. Dobran, M., Nasi, D., Brunozzi, D., di Somma, L., Gladi, M., lacoangeli, M., \& Scerrati, M. (2016). Treatment of unstable thoracolumbar junction fractures: short-segment pedicle fixation with inclusion of the fracture level versus long-segment instrumentation. Acta neurochirurgica, 158(10), 1883-9. doi:10.1007/s00701-016-2907-0

12. Joaquim, A. F., de Almeida Bastos, D. C., Jorge Torres, H. H., \& Patel, A. A. (2016). Thoracolumbar Injury Classification and Injury Severity Score System: A Literature Review of Its Safety. Global spine journal, 6(1), 80-5. doi:10.1055/s-0035-1554775

13. Alanay, A., Acaroglu, E., Yazici, M., Oznur, A., \& Surat, A. (2001). Short-segment pedicle instrumentation of thoracolumbar burst fractures: does transpedicular intracorporeal grafting prevent early failure? Spine, 26(2), 213-7.

14. Ökten, A. İ., Gezercan, Y., Özsoy, K. M., Ateş, T., Menekşe, G., Aslan, A., . . Güzel, A. (2015). Results of treatment of unstable thoracolumbar burst fractures using pedicle instrumentation with and without fracture-level screws. Acta neurochirurgica, 157(5), 831-6. doi:10.1007/s00701-015-2383-y

15. Wu, H., Zhao, D. X., Jiang, R., \& Zhou, X. Y. (2016). Surgical treatment of Denis type B thoracolumbar burst fracture with neurological deficiency by paraspinal approach. Braz J Med Biol Res, 49(11), e5599. doi:10.1590/1414-431X20165599

16. Stancić, M. F., Gregorović, E., Nozica, E., \& Penezić, L. (2001). Anterior decompression and fixation versus posterior reposition and semirigid fixation in the treatment of unstable burst thoracolumbar fracture: prospective clinical trial. Croatian medical journal, 42(1), 49-53.

17. Waqar, M., Van-Popta, D., Barone, D. G., Bhojak, M., Pillay, R., \& Sarsam, Z. (2017). Short versus longsegment posterior fixation in the treatment of thoracolumbar junction fractures: a comparison of outcomes. Br J Neurosurg, 31(1), 54-7. doi:10.1080/02688697.2016.1206185

18. Mahar, A., Kim, C., Wedemeyer, M., Mitsunaga, L., Odell, T., Johnson, B., \& Garfin, S. (2007). Shortsegment fixation of lumbar burst fractures using pedicle fixation at the level of the fracture. Spine, 32(14), 1503-07. 
19. Liao, J.-C., Chen, W.-P., \& Wang, H. (2017). Treatment of thoracolumbar burst fractures by shortsegment pedicle screw fixation using a combination of two additional pedicle screws and vertebroplasty at the level of the fracture: a finite element analysis. BMC Musculoskelet Disord, 18(1), 262. doi:10.1186/s12891-017-1623-0

20. Norton, R. P., Milne, E. L., Kaimrajh, D. N., Eismont, F. J., Latta, L. L., \& Williams, S. K. (2014).

Biomechanical analysis of four- versus six-screw constructs for short-segment pedicle screw and rod instrumentation of unstable thoracolumbar fractures. The Spine Journal, 14(8), 1734-9. doi:https://doi.org/10.1016/j.spinee.2014.01.035

21. Tong, M.-J., Tang, Q., Wang, C.-G., Xiang, G.-H., Chen, Q., Xu, H.-Z., \& Tian, N.-F. (2018). Efficacy of Using Intermediate Screws in Short-Segment Fixation for Thoracolumbar Fractures: A Meta-Analysis of Randomized Controlled Trials. World Neurosurgery, 110, e271-e80. doi:https://doi.org/10.1016/j.wneu.2017.10.157

22. Müller, U., Berlemann, U., Sledge, J., \& Schwarzenbach, O. (1999). Treatment of thoracolumbar burst fractures without neurologic deficit by indirect reduction and posterior instrumentation: bisegmental stabilization with monosegmental fusion. European spine journal : official publication of the European Spine Society, the European Spinal Deformity Society, and the European Section of the Cervical Spine Research Society, 8(4), 284-9. doi:10.1007/s005860050175

23. Daniaux, H. (1986). [Transpedicular repositioning and spongioplasty in fractures of the vertebral bodies of the lower thoracic and lumbar spine]. Der Unfallchirurg, 89(5), 197-213.

24. Grabowski, G., \& Cornett, C. A. (2013). Bone graft and bone graft substitutes in spine surgery: current concepts and controversies. The Journal of the American Academy of Orthopaedic Surgeons, 21(1), 51-60. doi:10.5435/JAAOS-21-01-51

25. Knop, C., Fabian, H. F., Bastian, L., \& Blauth, M. (2001). Late results of thoracolumbar fractures after posterior instrumentation and transpedicular bone grafting. Spine, 26(1), 88-99.

26. Verlaan, J. J., Somers, I., Dhert, W. J., \& Oner, F. C. (2015). Clinical and radiological results 6 years after treatment of traumatic thoracolumbar burst fractures with pedicle screw instrumentation and balloon assisted endplate reduction. Spine J, 15(6), 1172-8. doi:10.1016/j.spinee.2013.11.044

\section{Figures}




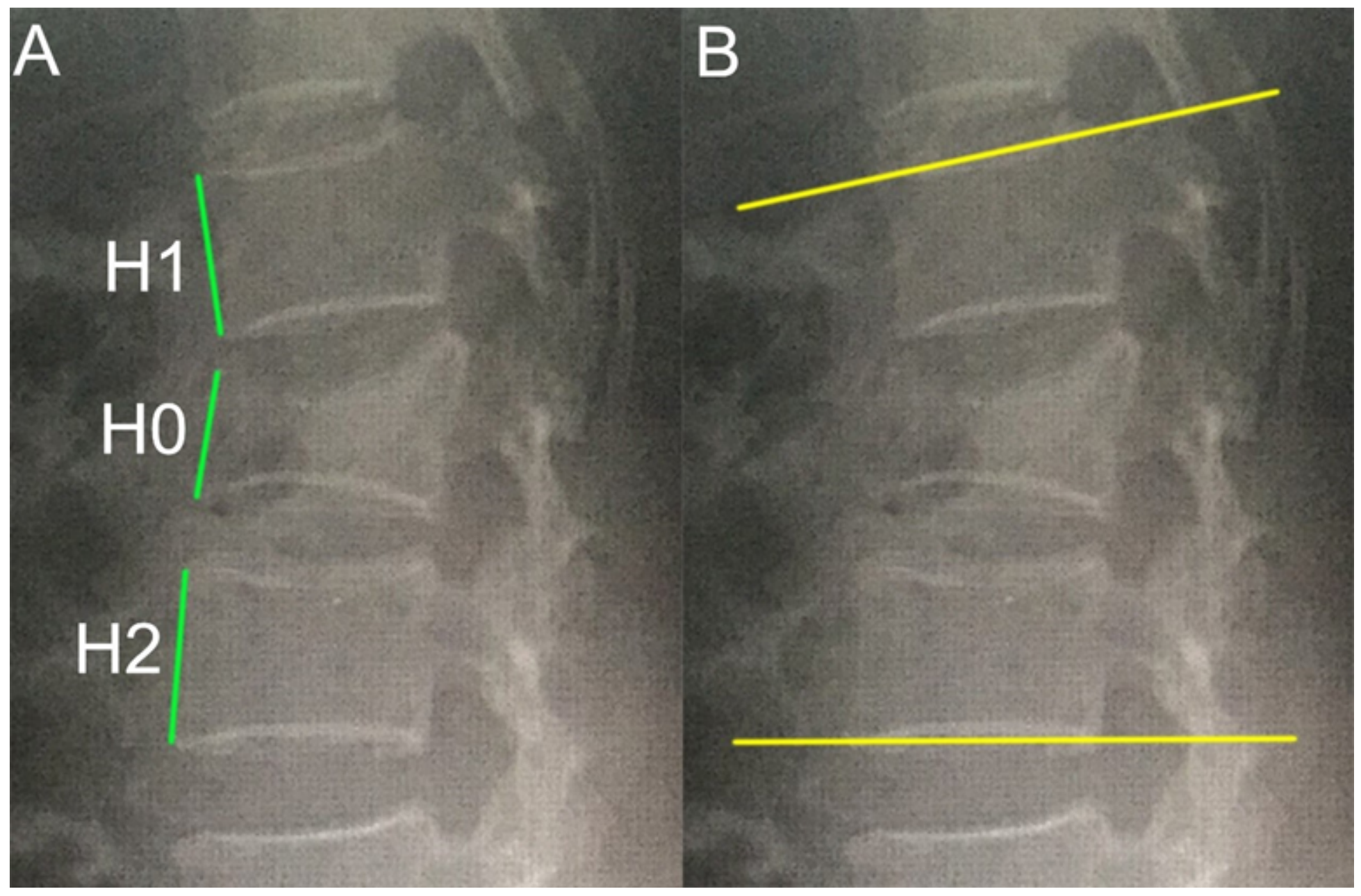

\section{Figure 1}

Schematic diagram of the methods for measuring the anterior vertebra height (AVH) and local kyphosis angle (LKA). (A) The measurements for the AVH is shown in the lateral radiographs. (B) The lateral radiographs showing measurements for the LKA. LKA was measured between the proximal end plates of the vertebra above the fractured vertebra and the distal end plates of the vertebra below the fractured vertebra (yellow line). $\mathrm{HO}, \mathrm{AVH}$ of injured vertebra; $\mathrm{H} 1, \mathrm{AVH}$ of superior vertebra adjacent to injured vertebra; $\mathrm{H} 2, \mathrm{AVH}$ of inferior vertebra adjacent to injured vertebra 


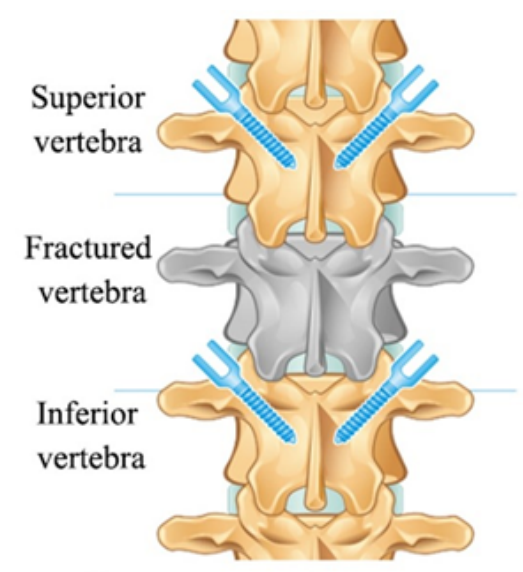

(1) Insert four pedicle screws into superior and inferior vertebra.

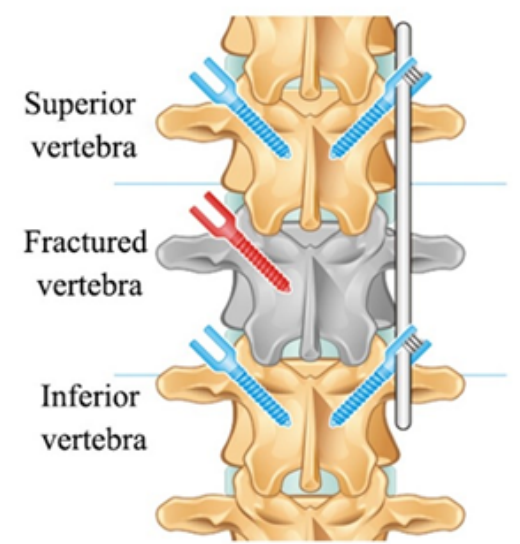

(3) Remove the rod of contralateral side and insert one pedicle screw into the fractured vertebra.

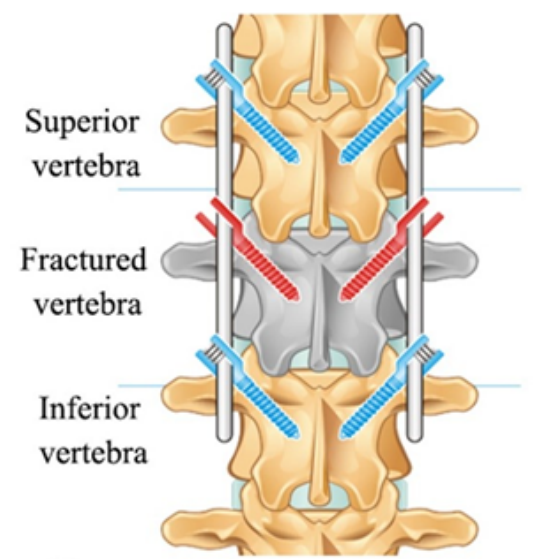

(5)Distract longitudinally again and tighten adjacent vertebra screws nuts.

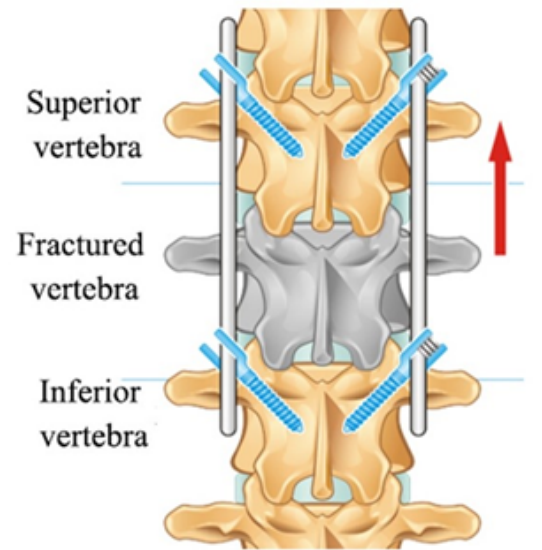

(2)Distract longitudinally and fix the rod on one side temporarily.

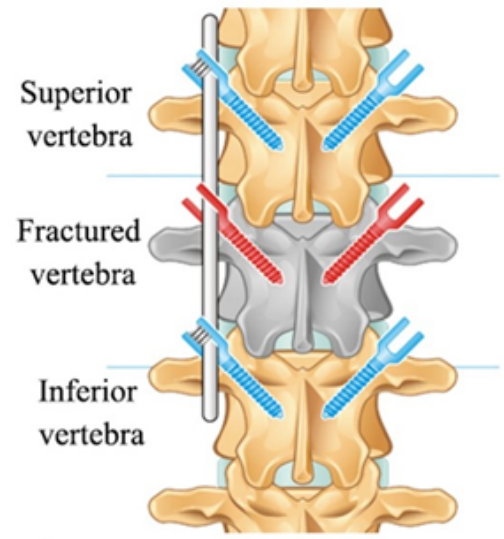

(4) Replace the rod, distract lengthwise and fix it.Then insert the pediclescrew on the other side in the same way.

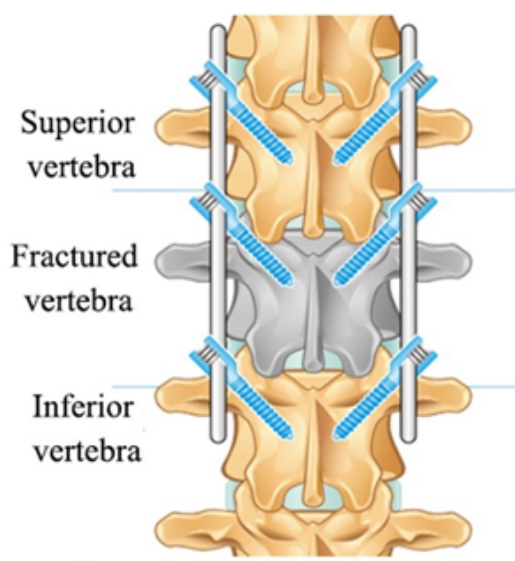

(6)Finally tighten the fractured vertebra screws nuts.

\section{Figure 2}

Schematic diagram of modified technique of posterior short-segment instrumentation 


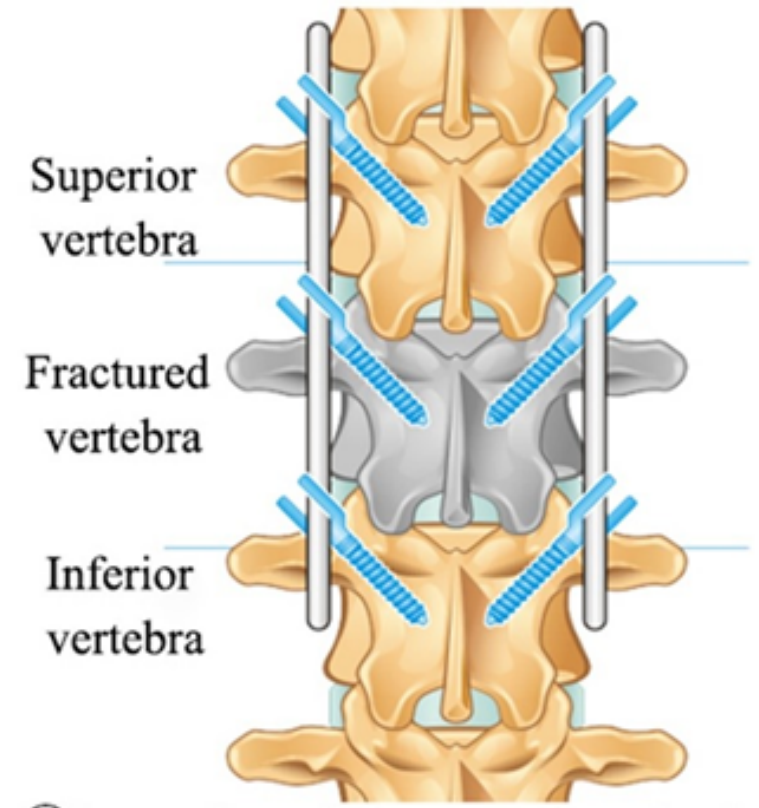

(1) Insert six pedicle screws into the fractured vertebra and one level above and below the fractured vertebra, then place the rods.

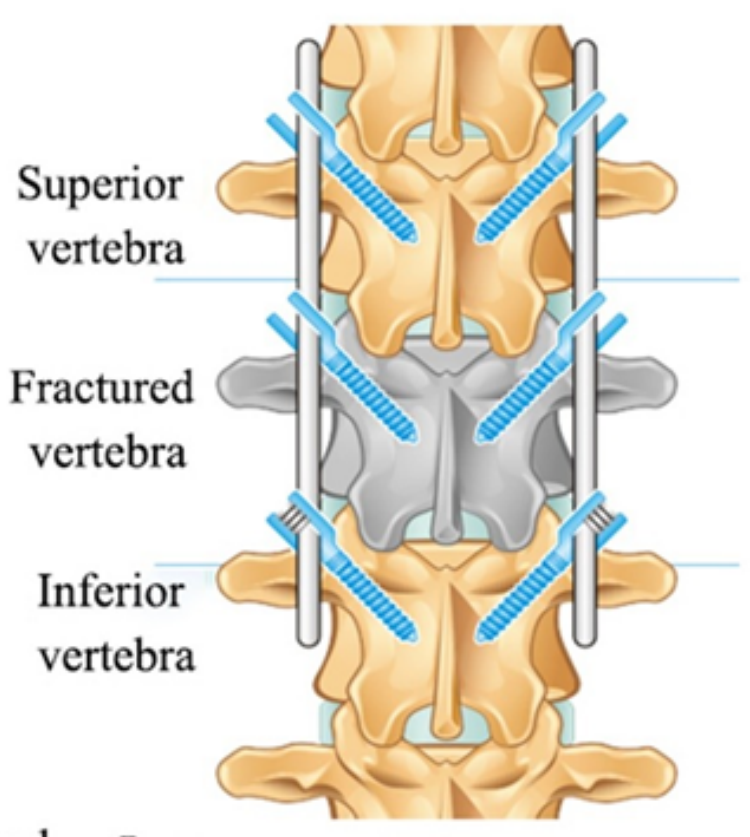

(2) Tighten the inferior vertebra screws nuts.

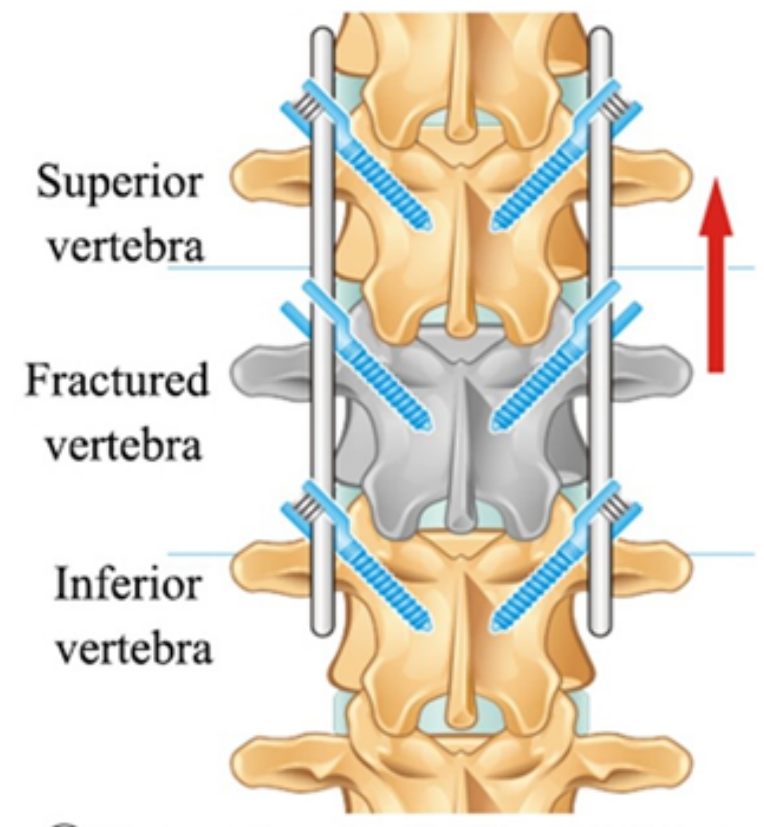

(3)Distract longitudinally and tighten the superior vertebra screws nuts.

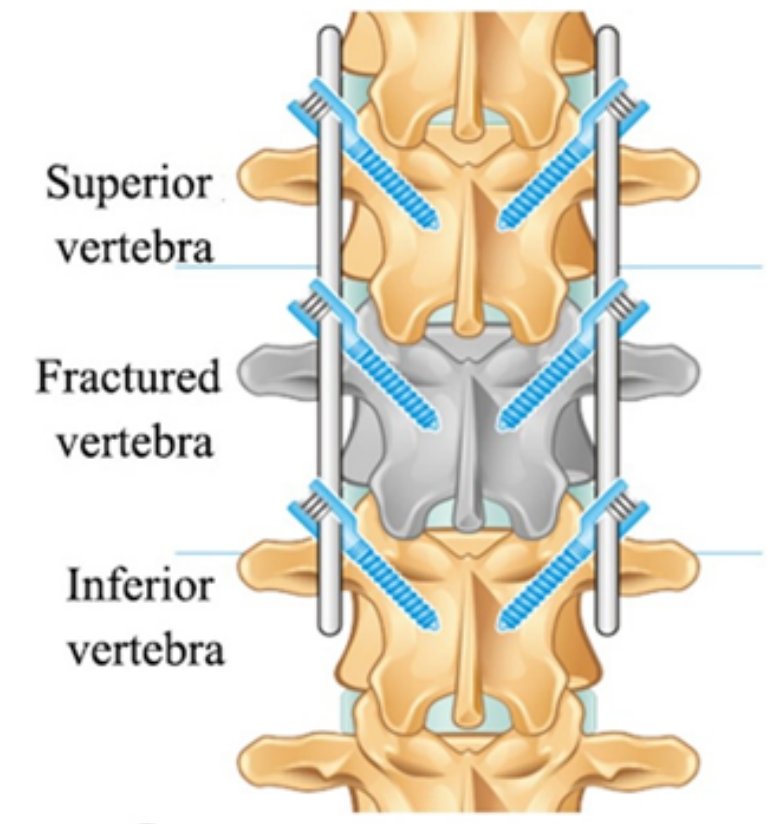

(4)Finally tighten the fractured vertebra screws nuts.

\section{Figure 3}

Schematic diagram of conventional technique of posterior short-segment instrumentation 


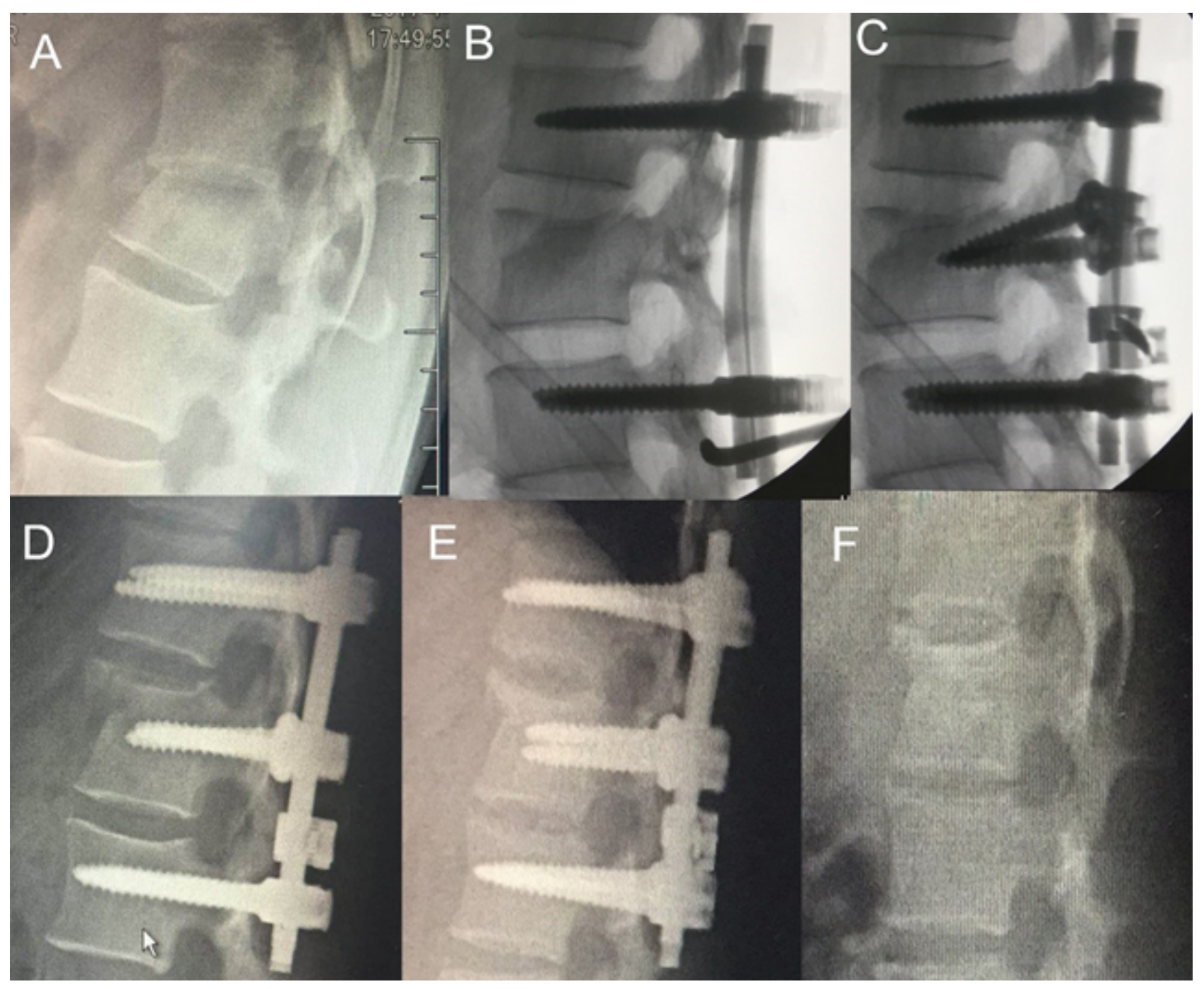

Figure 4

Radiographs of a 30-year-old woman with a lumbar burst fracture of the L1 vertebra with a Thoracolumbar Injury Classification and Severity score of 8 points. (A) An anterior vertebral height (AVH) ratio of $63.6 \%$ and local kyphosis angle (LKA) of $9^{\circ}$ were shown in preoperative lateral radiograph. (B) Intraoperative lateral radiograph showed that we placed four pedicle screws into the superior and inferior vertebrae firstly. Then we connected the rods and screws temporarily and performed distraction longitudinally. (C) Intraoperative lateral radiograph showed the pedicle screws were placed into the fractured vertebra and short-segment pedicle instrumentation was completed finally. (D) The AVH ratio and LKA were corrected to $103.0 \%$ and $6^{\circ}$ respectively as is shown in radiograph at 1 week after surgery. (E) An AVH ratio of $102.1 \%$ and LKA of $4^{\circ}$ were shown in radiograph at 1 -year follow up, suggesting no loss in the AVH ratio or LKA. (F) An ABH ratio of $98.7 \%$ and LKA of $5^{\circ}$ were shown in radiograph at 6 months after implant removal, suggesting no obvious loss of $A V H$ ratio or LKA. 


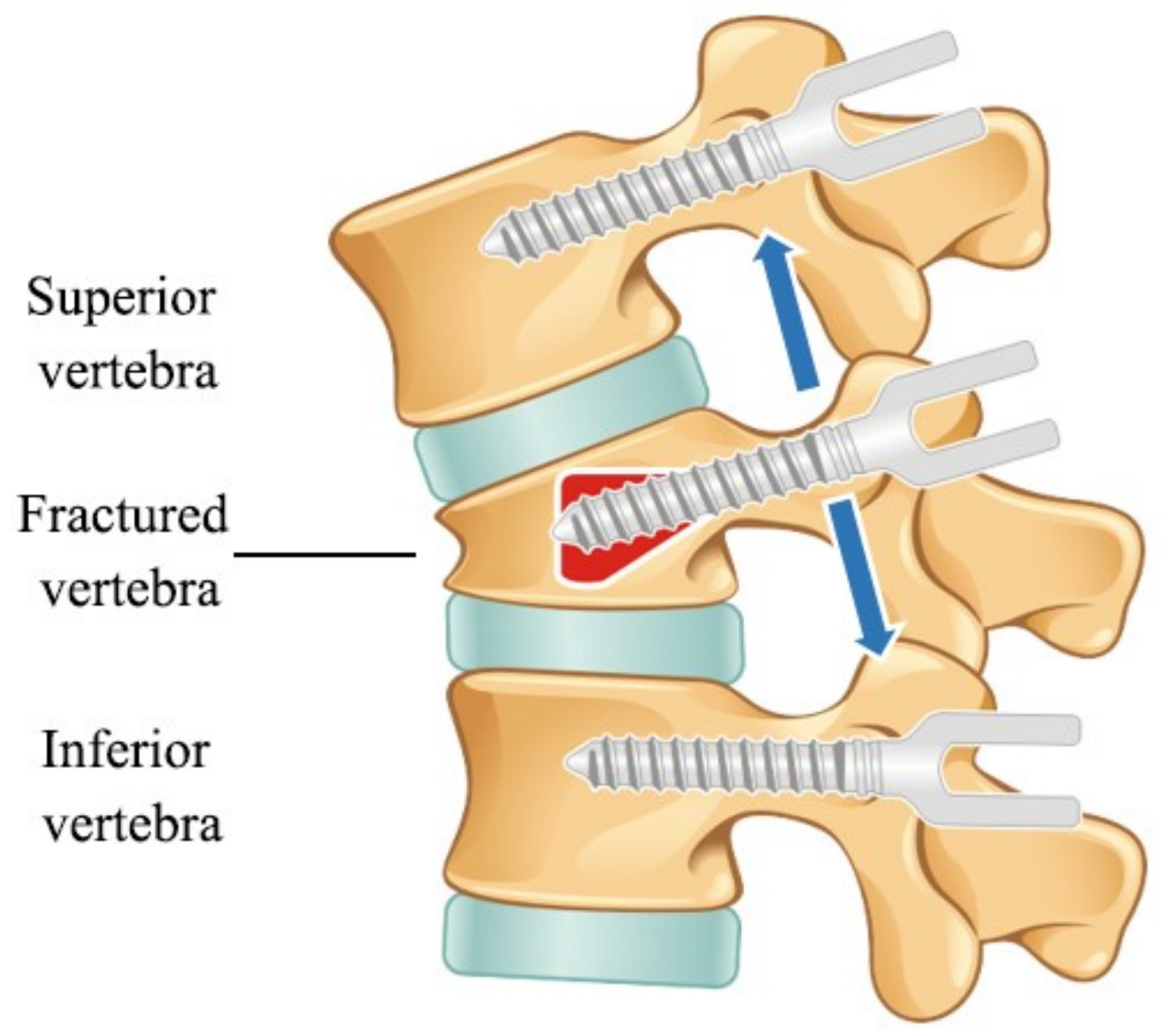

Figure 5

Swing and cutting effect of fractured vertebral screws in the process of distraction
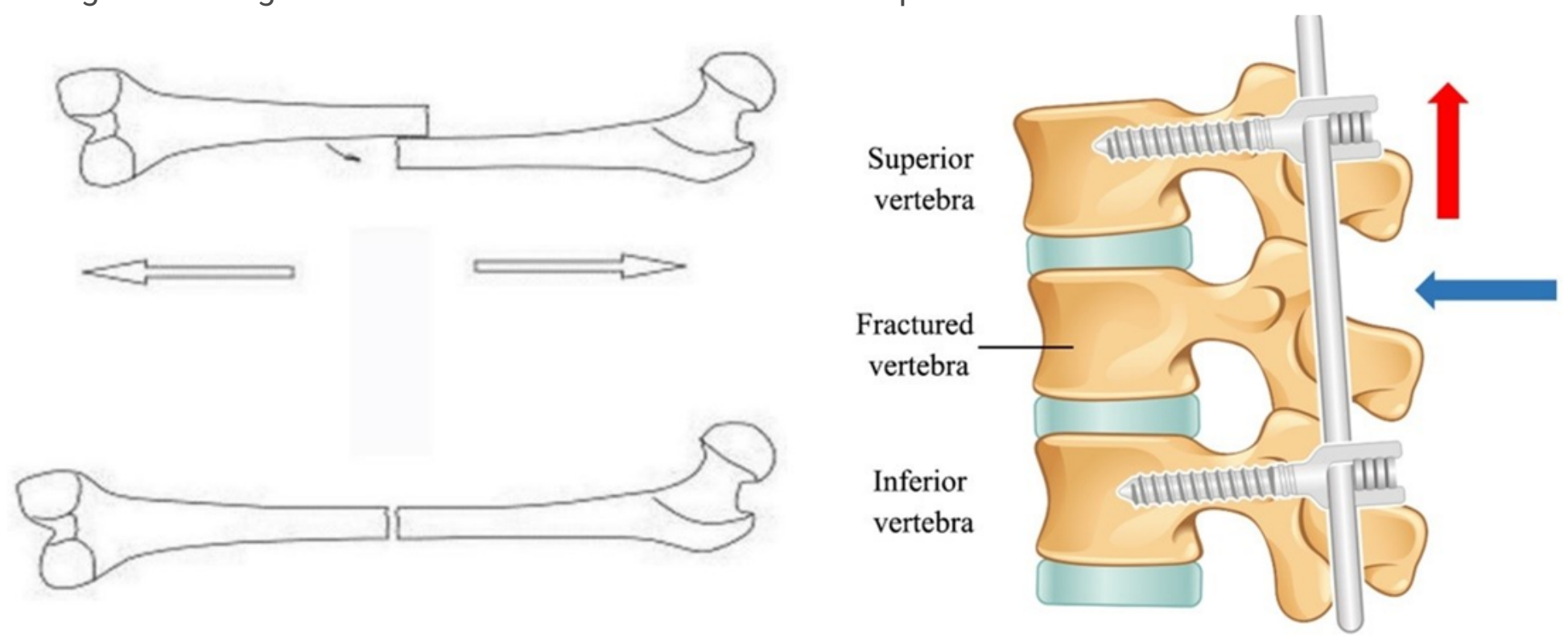

Figure 6

Combination of longitudinal continuous traction force and transverse push extrusion force. 